\title{
315.
}

\section{ON THE CUBIC CENTRES OF A LINE WITH RESPECT TO THREE LINES AND A LINE.}

[From the Philosophical Magazine, vol. xxII. (1861), pp. 433-436.]

ON referring to my Note on this subject (Phil. Mag. vol. xx. pp. 418-423, 1860 [257]), it will be seen that the cubic centres of the line

$$
\lambda x+\mu y+\nu z=0
$$

in relation to the lines $x=0, y=0, z=0$, and the line $x+y+z=0$, are determined by the equations

$$
x: y: z=\frac{1}{\theta+\lambda}: \frac{1}{\theta+\mu}: \frac{1}{\theta+\nu},
$$

where $\theta$ is a root of the cubic equation

$$
\frac{1}{\theta+\lambda}+\frac{1}{\theta+\mu}+\frac{1}{\theta+\nu}-\frac{2}{\theta}=0
$$

or as it may also be written,

$$
\theta^{3}-\theta(\mu \nu+\nu \lambda+\lambda \mu)-2 \lambda \mu \nu=0 .
$$

Two of the centres will coincide if the equation for $\theta$ has equal roots; and this will be the case if

$$
\lambda^{-\frac{1}{3}}+\mu^{-\frac{1}{3}}+\nu^{-\frac{1}{3}}=0,
$$

or, what is the same thing, if $\lambda, \mu, \nu=a^{-3}, b^{-3}, c^{-3}$, where $a+b+c=0$. In fact, if $a+b+c=0$, then $a^{3}+b^{3}+c^{3}=3 a b c$, and the equation in $\theta$ becomes

$$
\theta^{3}-\frac{3 \theta}{a^{2} b^{2} c^{2}}-\frac{2}{a^{3} b^{3} c^{3}}=0
$$

c. v. 
that is

which is

$$
(a b c \theta)^{3}-3(a b c \theta)-2=0
$$

$$
(a b c \theta+1)^{2}(a b c \theta-2)=0 ;
$$

so that the values of $\theta$ are $\frac{-1}{a b c}, \frac{2}{a b c}$.

First, if $\theta=-\frac{1}{a b c}$, then $x, y, z$ will be the coordinates of the double centre. And we have

$$
\theta+\lambda=\frac{1}{a^{3}}-\frac{1}{a b c}=\frac{1}{2 a^{3} b c}\left(2 b c-2 a^{2}\right)=\frac{1}{2 a^{3} b c}\left(-a^{2}-b^{2}-c^{2}\right) ;
$$

or putting for shortness $\square=a^{2}+b^{2}+c^{2}$,

$$
\theta+\lambda=-\frac{1}{2 a^{3} b c} \square, \quad=-\frac{3}{a b c} \cdot \frac{\square}{6 a^{2}}
$$

with similar values for $\theta+\mu, \theta+\nu$. But $\frac{1}{x}, \frac{1}{y}, \frac{1}{z}$ are proportional to $\theta+\lambda, \theta+\mu, \theta+\nu$; and we may therefore write

$$
\frac{P}{x}=\frac{\square}{6 a^{2}}, \quad \frac{P}{y}=\frac{\square}{6 b^{2}}, \quad \frac{P}{z}=\frac{\square}{6 c^{2}} ;
$$

whence, in virtue of the equation $a+b+c=0$, we have for the locus of the double centre,

$$
\sqrt{x}+\sqrt{y}+\sqrt{z}=0
$$

or this locus is a conic touching the lines $x=0, y=0, z=0$ harmonically in respect to the line $x+y+z=0$, a result which was obtained somewhat differently in the paper above referred to.

Next, if $\theta=\frac{2}{a b c}, x, y, z$ will be the coordinates of the single centre. And we now have

$$
\theta+\lambda=\frac{1}{a^{3}}+\frac{2}{a b c}=\frac{1}{2 a^{3} b c}\left(2 b c-2 a^{2}+6 a^{2}\right)=\frac{1}{2 a^{3} b c}\left(-\square+6 a^{2}\right)=-\frac{3}{a b c} \frac{\square-6 a^{2}}{6 a^{2}}
$$

with similar values for $\theta+\mu, \theta+\nu$. But $\frac{1}{x}, \frac{1}{y}, \frac{1}{z}$ are proportional to $\theta+\lambda, \theta+\mu, \theta+\nu$, and we may therefore write

$$
\frac{P}{x}=\frac{\square-6 a^{2}}{6 a^{2}}, \frac{P}{y}=\frac{\square-6 b^{2}}{6 b^{2}}, \quad \frac{P}{z}=\frac{\square-6 c^{2}}{6 c^{2}},
$$

from which equations, and the equation $a+b+c=0$, the quantities $P, a, b, c$ have to be eliminated. I at first effected the elimination as follows: viz., writing the equations under the form

$$
\frac{x}{x+P}=\frac{6 a^{2}}{\square}, \quad \frac{y}{y+P}=\frac{6 b^{2}}{\square}, \frac{z}{z+P}=\frac{6 c^{2}}{\square},
$$


we obtain

$$
\begin{gathered}
\frac{x}{x+P}+\frac{y}{y+P}+\frac{z}{z+P}=6 \\
\sqrt{\frac{x}{x+P}}+\sqrt{\frac{y}{y+P}}+\sqrt{\frac{z}{z+P}}=0
\end{gathered}
$$

which are easily transformed into

$$
\begin{gathered}
\frac{x}{x+P}+\frac{y}{y+P}+\frac{z}{z+P}=6, \\
\frac{y z}{(y+P)(z+P)}+\frac{z x}{(z+P)(x+P)}+\frac{x y}{(x+P)(y+P)}=9 ;
\end{gathered}
$$

or, what is the same thing,

$$
\begin{aligned}
& 6(P+x)(P+y)(P+z)-x(P+y)(P+z)-y(P+z)(P+x)-z(P+x)(P+y)=0, \\
& 9(P+x)(P+y)(P+z)-y z(P+x) \quad-z x(P+y) \quad-x y(P+z)=0,
\end{aligned}
$$

which give

$$
\begin{aligned}
& 6 P^{3}+5 P^{2}(x+y+z)+4 P(y z+z x+x y)+3 x y z=0, \\
& 9 P^{3}+9 P^{2}(x+y+z)+8 P(y z+z x+x y)+6 x y z=0
\end{aligned}
$$

or, multiplying the first equation by 2 , and subtracting the second,

$$
3 P+(x+y+z)=0 ;
$$

and we thus obtain for the locus of the single centre the equation

$$
\frac{x}{-2 x+y+z}+\frac{y}{-2 y+z+x}+\frac{z}{-2 z+x+y}=2,
$$

or, what is the same thing,

$$
x^{3}+y^{3}+z^{3}-\left(y z^{2}+z x^{2}+x y^{2}+y^{2} z+z^{2} x+x^{2} y\right)+3 x y z=0,
$$

which may also be written,

$$
-(-x+y+z)(x-y+z)(x+y-z)+x y z=0 .
$$

The same result may also be obtained as follows: viz, observing that

we have

$$
\square-6 a^{2}=b^{2}+c^{2}-5 a^{2}=-4 a^{2}-2 b c,
$$

$$
\frac{x}{P}=\frac{-3 a^{2}}{2 a^{2}+b c}, \quad \frac{y}{P}=\frac{-3 b^{2}}{2 b^{2}+c a}, \quad \frac{z}{P}=\frac{-3 c^{2}}{2 c^{2}+a b},
$$

and then by means of the equation

$$
\frac{a^{2}}{2 a^{2}+b c}+\frac{b^{2}}{2 b^{2}+a c}+\frac{c^{2}}{2 c^{2}+a b}-1=0,
$$


which is identically true in virtue of $a+b+c=0$ (in fact, multiplying out, this gives

$$
\begin{array}{r}
12 a^{2} b^{2} c^{2}+4\left(b^{3} c^{3}+c^{3} a^{3}+a^{3} b^{3}\right)+a b c\left(a^{3}+b^{3}+c^{3}\right) \\
-8 a^{2} b^{2} c^{2}-4\left(b^{3} c^{3}+c^{3} a^{3}+a^{3} b^{3}\right)-2 a b c\left(a^{3}+b^{3}+c^{3}\right)-a^{2} b^{2} c^{2}=0 ;
\end{array}
$$

that is

$$
3 a^{2} b^{2} c^{2}-a b c\left(a^{3}+b^{3}+c^{3}\right)=0 \text {, or } a b c\left(a^{3}+b^{3}+c^{3}-3 a b c\right)=0,
$$

where the second factor divides by $a+b+c$ ), we find the above-mentioned equation,

We then have

$$
x+y+z+3 P=0 .
$$

$$
\frac{-x+y+z}{P}=\frac{x+y+z}{P}-\frac{2 x}{P}=-3+\frac{6 a^{2}}{2 a^{2}+b c}=-\frac{3 b c}{2 a^{2}+b c}
$$

that is

$$
\frac{-x+y+z}{P}=\frac{-3 b c}{2 a^{2}+b c}, \quad \frac{x-y+z}{P}=\frac{-3 c a}{2 b^{2}+c}, \quad \frac{x+y-z}{P}=\frac{-3 a b}{2 c^{2}+a b}
$$

and forming the product of these functions, and that of the foregoing values of $\frac{x}{P}, \frac{y}{P}, \frac{z}{P}$, we find as before,

$$
-(-x+y+z)(x-y+z)(x+y-z)+x y z=0
$$

for the equation of the locus of the single centre. The equation shows that the locus is a cubic curve which touches the lines $x=0, y=0, z=0$ at the points where these lines are intersected by the lines $y-z=0, z-x=0, x-y=0$ (that is, it touches the lines $x=0, y=0, z=0$ harmonically in respect to the line $x+y+z=0$ ), and besides meets the same lines $x=0, y=0, z=0$ at the points in which they are respectively met by the line $x+y+z=0$.

2, Stone Buildings, W.C., September 25, 1861. 